\title{
Insights into the mechanism(s) of von Willebrand factor degradation during mechanical circulatory support
}

\author{
Carlo R. Bartoli, MD, PhD, ${ }^{\mathrm{a}, \mathrm{b}}$ Sujith Dassanayaka, MS, ${ }^{\mathrm{c}, \mathrm{e}}$ Kenneth R. Brittian, BS, ${ }^{\mathrm{c}}$ Andrew Luckett, MD, ${ }^{\mathrm{d}}$ \\ Srinivas Sithu, PhD, ${ }^{\mathrm{e}}$ Thorsten Siess, PhD, ${ }^{\mathrm{f}}$ Daniel H. Raess, MD, ${ }^{\mathrm{f}}$ Paul A. Spence, MD, ${ }^{\mathrm{g}}$ \\ Steven C. Koenig, PhD, ${ }^{\text {h,i }}$ Robert D. Dowling, MD, ${ }^{j}$ and Stanley E. D’Souza, $\mathrm{PhD}^{\mathrm{e}}$
}

Objective: Left ventricular assist device support produces a bleeding diathesis. Evidence suggests a major role for von Willebrand factor (vWF). We examined vWF metabolism in a preclinical model of short-term mechanical circulatory support.

\begin{abstract}
Methods: In 25 calves (weight, 80-110 kg), the inflow/outflow graft of the Symphony Heart Assist System was sewn end-to-side to the carotid artery. Support was initiated (acute, $n=4 ; 1$ week, $n=16 ; 2$ weeks, $n=5$ ). Acutely, carotid artery pressure and flow were measured to evaluate the hemodynamic changes near the anastomosis. At baseline and after $\leq 2$ weeks of support, platelet aggregometry with adenosine $5^{\prime}$-diphosphate, collagen, and ristocetin was performed. Gel electrophoresis and wet immunoblotting qualitatively evaluated vWF multimers and quantified plasma ADAMTS-13, the vWF-cleaving protease. Carotid arterial rings near the anastomosis were studied with immunohistochemical staining for ADAMTS-13 and were cultured to quantify endothelial ADAMTS-13 production. Fluorescent resonance energy transfer was used to evaluate the enzymatic activity of ADAMTS-13 in the plasma and in supernatant from cultured carotid arterial rings. Plasma interleukin-6, which inhibits ADAMTS-13 activity, was measured using an enzyme-linked immunosorbent assay.
\end{abstract}

Results: During support, statistically significant $(P<.05)$ changes in the carotid endothelium arterial hemodynamics were observed. The highest molecular weight vWF multimers were absent, and the vWF-ristocetin platelet aggregation pathway was significantly impaired. A modest but significant increase in plasma ADAMTS-13 protein and activity was observed. ADAMTS-13 decreased significantly in the carotid near the anastomosis but increased significantly in supernatant from cultured carotid arterial rings. The plasma interleukin-6 levels did not change significantly.

Conclusions: Hemodynamic activation of vWF and increased plasma ADAMTS-13 activity may have reduced high-molecular-weight vWF multimers and thereby impaired the vWF-platelet aggregation pathway. Additional delineation of these pathways may improve management of left ventricular assist device-associated bleeding. (J Thorac Cardiovasc Surg 2014;147:1634-43)

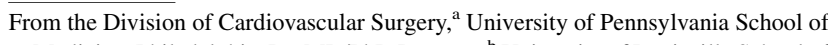
Medicine, Philadelphia, Pa; MD/PhD Program, ${ }^{\text {b }}$ University of Louisville School of Medicine, Louisville, Ky; Institute of Molecular Cardiology, ${ }^{\mathrm{c}}$ University of Louisville School of Medicine, Louisville, Ky; University of Louisville School of Medicine, ${ }^{\mathrm{d}}$ Louisville, Ky; Department of Physiology and Biophysics, ${ }^{\mathrm{e}}$ University of Louisville School of Medicine, Louisville, Ky; Abiomed Inc, ${ }^{\mathrm{f}}$ Danvers, Mass; SCR Inc, ${ }^{,}$Louisville, Ky; Cardiovascular Innovation Institute, ${ }^{\mathrm{h}}$ University of Louisville School of Medicine, Louisville, Ky; Department of Bioengineering, ${ }^{i}$ University of Louisville, Louisville, Ky; and Dowling Consulting, PSC, ${ }^{\mathrm{j}}$ Louisville, Ky.

Funding for this project was provided by the National Institutes of Health grant HL43721 National Institutes of Health Small Business Innovation Research grants 2R44HL083586-02A1, 2R44HL088760-02, and R43HL102981, and Kentucky Science and Technology Corporation grants KSTC-184-512-08-054 and KSTC184-512-08-054.

Disclosures: Drs Siess and Raess are paid employees of Abiomed, Inc. Dr Spence receives royalties for the Symphony pump. Dr Dowling is a paid consultant of Abiomed, Inc. All other authors have nothing to disclose with regard to commercial support.

Received for publication May 21, 2013; revisions received July 19, 2013; accepted for publication Aug 16, 2013; available ahead of print Oct 17, 2013.

Address for reprints: Stanley E. D'Souza, PhD, Department of Physiology and Biophysics, University of Louisville School of Medicine, 500 S Preston St, HSC A 1115, Louisville, KY 40292 (E-mail: sedsou01@louisville.edu).

$0022-5223 / \$ 36.00$

Copyright $(9) 2014$ by The American Association for Thoracic Surgery

http://dx.doi.org/10.1016/j.jtcvs.2013.08.043
Recent reports have documented impairment of the von Willebrand factor (vWF) pathway ${ }^{1-11}$ and nonsurgical bleeding ${ }^{12}$ during mechanical circulatory support. In these studies, acquired von Willebrand syndrome developed in patients with continuous-flow ${ }^{1-11}$ and pulsatile-flow ${ }^{1,4,5,9}$ left ventricular assist devices (LVADs). The absence of high-molecular-weight vWF multimers contributed to a bleeding diathesis in these patients.

Limited data exist to explain the mechanisms of acquired von Willebrand syndrome in patients with an LVAD. During in vitro LVAD support in a mock circulatory loop, degradation of vWF occurred rapidly as a result of multiple mechanisms that occurred simultaneously. ${ }^{13}$ Shearinduced mechanical demolition, in which vWF monomer subunits were broken off from vWF multimers, may have played a significant role. In parallel, ADAMTS-13, the vWF protease, constitutively cleaved vWF into fragments in the plasma.

To investigate the potential pathophysiologic mechanisms of acquired von Willebrand syndrome during mechanical circulatory support in vivo, we implanted the Symphony Heart 

Abbreviations and Acronyms
$\mathrm{ADP}=$ adenosine $5^{\prime}$-diphosphate
FRET $=$ fluorescent resonance energy transfer
IL $\quad=$ interleukin
LVAD $=$ left ventricular assist device
PRP = platelet-rich plasma
SDS = sodium dodecyl sulfate
$\mathrm{vWF}=$ von Willebrand factor

Assist System ${ }^{14}$ (Abiomed, Inc, Danvers, Mass), a novel partial-support counterpulsatile LVAD, in calves. Platelet aggregation, vWF multimers, ADAMTS-13, and interleukin (IL)-6, which inhibits ADAMTS-13, were examined.

\section{METHODS}

The present study was conducted in accordance with the National Institutes of Health guidelines for the care and use of animals in research. The Institutional Animal Care and Use Committee of the University of Louisville (Louisville, Ky) approved all experimental procedures.

\section{Study Overview}

The Symphony Heart Assist System (Abiomed, Inc) is a $30-\mathrm{mL}$ displacement pump designed to deliver prolonged partial support in ambulatory patients with heart failure (Figure 1, $B$ and $C$ ). The pump is implanted posterior to the pectoralis major muscle without entering the chest. A modified Gore-Tex graft (W. L. Gore \& Associates, Flagstaff, Ariz) is sewn end-to-side to the subclavian artery. The patient's electrocardiogram is continuously monitored, and an R-wave-sensing algorithm controls filling and emptying of the pump. The Symphony fills during native cardiac systole to reduce left ventricular afterload and ejects during native cardiac diastole to augment the diastolic blood pressure. Improved coronary and systemic blood flow occurs. By these mechanisms, the myocardial oxygen supply/demand relationship improves, ${ }^{14}$ and endorgan perfusion increases.

Aspects of the design, implantation strategy, support mechanism, and control algorithm of the Symphony pump are unique. The Symphony is designed with a valveless inflow/outflow cannula. A constant but shifting vortex continuously "washes" the interior of the pump to prevent thrombosis. Implantation (and explantation) with a simple subcutaneous procedure does not require extracorporeal circulation, sternotomy, or thoracotomy. The Symphony functions as a peripheral capacitance chamber that does not influence the internal impedance of the aorta. Adjustment of the timing of filling and ejection permit modest tradeoffs between improved coronary flow and reduced left ventricular workload. Consequently, subtle variations in the delivery of support may have important utility for incremental patient management on an individualized basis, especially during weaning of the device if explantation is indicated. Importantly, the low manufacturing cost and cost of implantation of the Symphony pump may expand the use of mechanical circulatory support in underserved foreign markets. A clinical trial with the Symphony pump is currently underway in Canada and France. ${ }^{15}$

We used 25 male calves (weight, $80-110 \mathrm{~kg}$ ) to evaluate the Symphony pump. The study was performed according to the Good Laboratory Practices (GLP) guidelines to determine the preclinical safety of the pump. The calves were supported acutely $(n=4)$ or were provided uninterrupted support for 1 week $(n=16)$ or 2 weeks $(n=5)$ with the Symphony pump.

The Symphony pump was implanted subcutaneously in the neck. An anastomosis was performed between the pump graft and the carotid artery as described below. This approach was chosen because of the similar size (approximately $8-10 \mathrm{~mm}$ in diameter) of the bovine carotid artery and the human subclavian artery and the similar distance (approximately $8-10 \mathrm{~cm}$ in distance) from the anastomosis to the aortic valve in humans and cows. ${ }^{16}$

\section{Experimental Design}

Blood samples were collected at baseline before implantation of the Symphony pump and after 1 or 2 weeks of support prior to euthanasia. As such, each calf was its own control. Likewise, analysis of the carotid artery harvested near the anastomosis of the pump and the contralateral carotid artery enabled each calf to also be used as its own control.

Platelet aggregometry was performed on platelet-rich plasma (PRP, $\mathrm{n}=14$ ) to evaluate platelet aggregation pathways. Gel electrophoresis and wet immunoblotting were performed to evaluate plasma vWF multimers $(\mathrm{n}=14)$ and to quantify plasma ADAMTS-13 $(\mathrm{n}=14)$. Immunohistochemical staining for ADAMTS-13 was performed on carotid arterial rings near the anastomosis $(n=8)$ to evaluate endothelial ADAMTS-13. Later, carotid arterial rings were cultured in vitro to quantify endothelial ADAMTS-13 production near the anastomosis $(n=5)$. Fluorescent resonance energy transfer (FRET) was used to evaluate plasma ADAMTS- 13 activity in the plasma $(\mathrm{n}=14)$ and in the supernatant from cultured carotid arterial rings $(n=5)$. An enzyme-linked immunosorbent assay was performed to measure plasma IL-6 $(n=14)$. In addition to the histologic and molecular analyses, carotid artery hemodynamics were measured acutely $(n=4)$ to quantify the hemodynamic changes associated with partial support using an end-to-side anastomosis to a peripheral artery.

\section{Surgical Preparation}

The calves were anesthetized with $3 \%$ to $5 \%$ isoflurane and prepared for sterile surgery. Permanent fluid-filled catheters were placed in the right jugular vein for intravenous access and in the proximal right carotid artery for arterial blood pressure monitoring. A left fifth intercostal space minithoracotomy was performed to place screw-in epicardial electrocardiographic leads (Medtronic, Inc, Minneapolis, Minn).

The calf was repositioned, and a left neck incision was made. The Symphony pump (30-mL stroke volume) was implanted in a subcutaneous pocket $(7 \mathrm{~cm} \times 7 \mathrm{~cm} \times 5 \mathrm{~cm})$ in the anterior neck. The percutaneous driveline was tunneled to the nape of the neck, externalized, and attached to a pneumatic driver (iPulse, Abiomed, Inc). A modified 8-mm Gore-Tex vascular graft (W. L. Gore \& Associates) was sewn end-to-side to the carotid artery and connected to the pump. Care was taken to completely de-air the pump. Pump filling and emptying were triggered by the electrocardiogram using R-wave recognition software. Device emptying and filling were timed to produce diastolic augmentation and afterload reduction, respectively.

In a subset of calves, carotid artery pressure and flow were measured acutely. Transit-time ultrasonic flow probes (Transonic, Inc, Ithaca, NY) were placed around the carotid artery proximally and distally to the anastomosis. High-fidelity single-tip micromanometer catheters (Millar Instruments, Inc, Houston, Tex) were placed directly into the carotid artery proximal and distal to the anastomosis. The Symphony was operated in an uninterrupted 1:1 support mode in which each ventricular systole resulted in pump filling and each ventricular diastole-triggered pump ejection. Hemodynamics were recorded for 30-s epochs at baseline and for 30-s epochs during 1:1 Symphony support.

Each calf was maintained on a daily dose of $75 \mathrm{mg}$ of clopidogrel, which was started 1 day before surgery. Intraoperatively, the calves received $10,000 \mathrm{U}$ of heparin before clamping the carotid artery to perform the end-to-side graft anastomosis. After anastomosis completion and initiation of device support, heparin was reversed with $100 \mathrm{mg}$ of protamine. At 6 hours after surgery, a continuous heparin infusion was initiated and titrated to maintain an activated clotting time of $>200 \mathrm{~s}$. Warfarin was initiated on postoperative day 3 and titrated to maintain an international normalized 

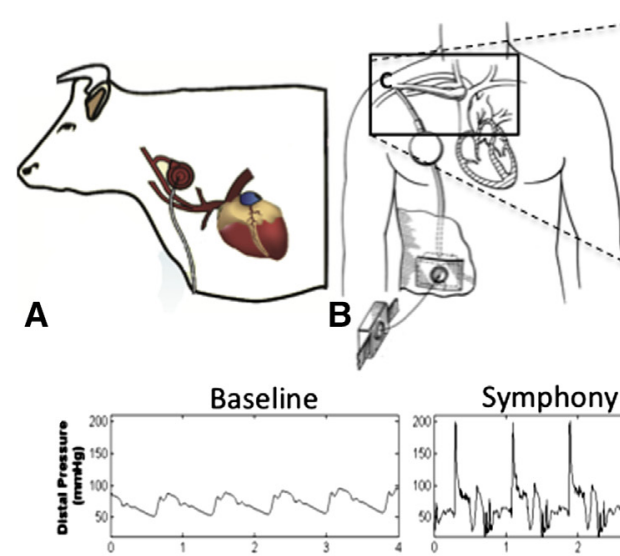

Symphony 1:1
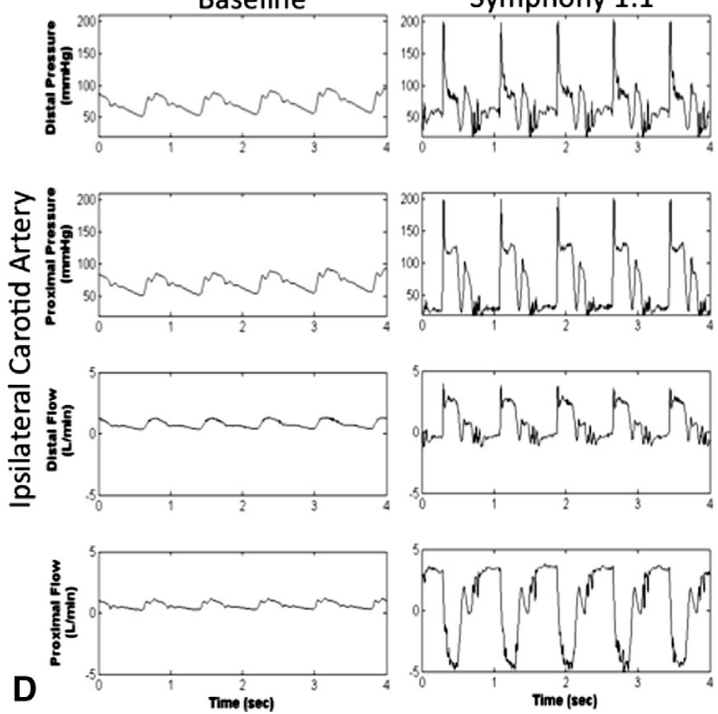

nime (sec)
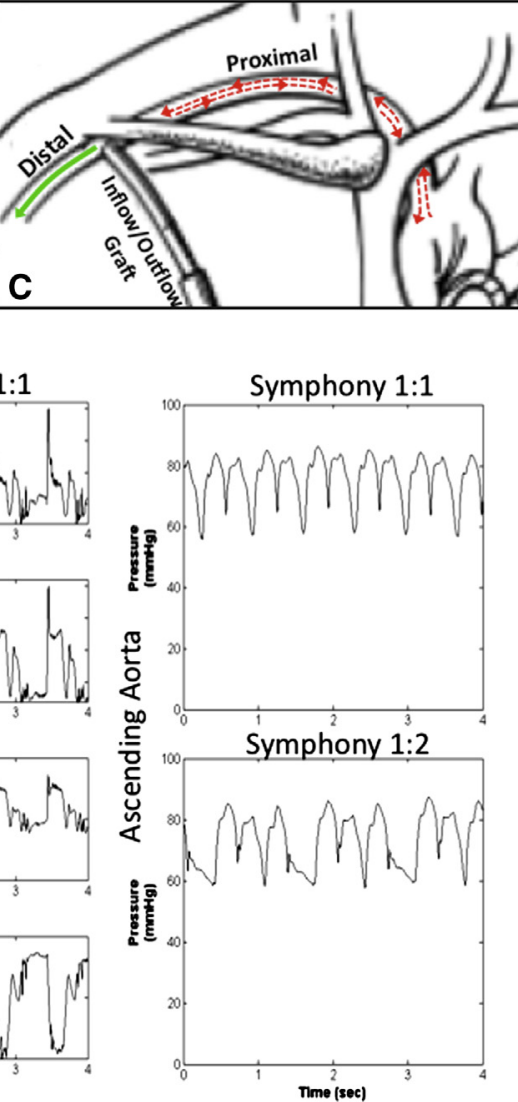
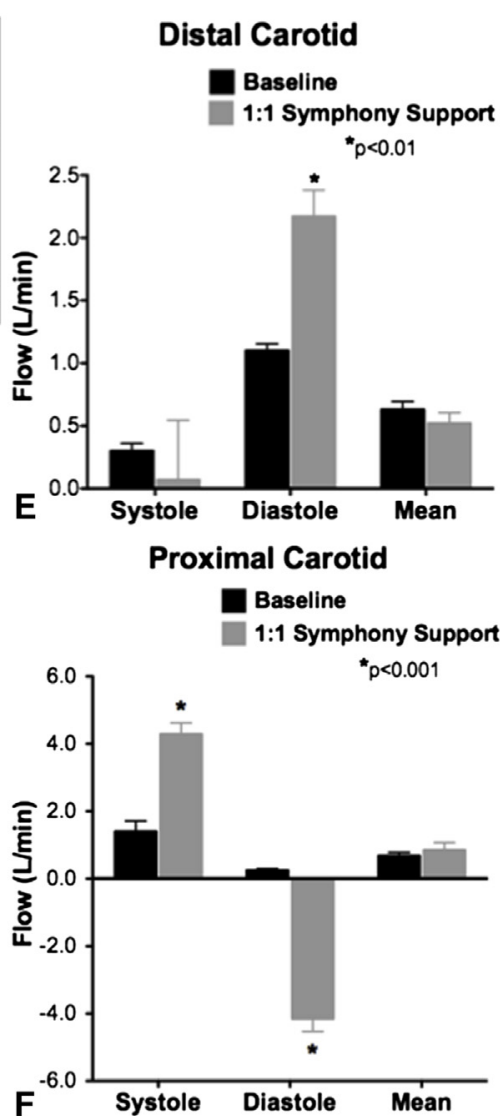

FIGURE 1. A, The anatomic configuration of the Symphony Heart Assist System is shown in cows. B and C, The anatomic configuration of the Symphony Heart Assist System is shown in humans. In humans, the pump is sewn end-to-side to the subclavian artery. The pump fills during native cardiac systole to reduce afterload and ejects during native cardiac diastole to augment diastolic blood pressure, increase coronary blood flow, and improve the myocardial oxygen supply/demand relationship. C and D, In the segment of artery between the anastomosis and the aorta, blood flows antegrade during pump filling and retrograde during pump ejection. Thus, the hemodynamic profile (pressure, flow, flow directionality, turbulence, shear stress) at the anastomosis is changed significantly. D, At baseline, a normal profile of carotid arterial blood pressures and flow were present (left). During 1:1 support, in which each ventricular systole triggered pump filling and each ventricular diastole triggered pump ejection, nonphysiologic pressures and flow were observed (middle). The aortic pressure tracings demonstrated effective diastolic augmentation during 1:1 support (top right) and 1:2 support (bottom right). E and F, Blood flow was quantified at baseline and during 1:1 support proximally and distally to the anastomosis. In the segment of vessel distal to the anastomosis, augmented flow was observed during native cardiac diastole when the pump ejected. In the segment of vessel proximal to the anastomosis, augmented flow was observed during native cardiac systole when the pump was filling. Complete flow reversal was observed in the artery proximal to the anastomosis during pump ejection.

ratio (INR) of 2.0 to 3.0. Heparin was discontinued after the INR was therapeutic.

After recovery, chronic animals underwent uninterrupted support for 1 or 2 weeks. On completion of the planned duration of support, euthanasia was induced with an intravenous bolus of Beuthanasia-D Special $(1 \mathrm{~mL} / 5 \mathrm{~kg})$.

\section{Hemodynamic Data Reduction and Analysis}

Hemodynamic transducers were pre- and postcalibrated against known physical standards to ensure measurement accuracy. High-fidelity hemodynamic waveforms were signal conditioned $(1000 \times$ gain and $60-\mathrm{Hz}$ low-pass filter) and analog-to-digital converted at a sampling rate of 400 $\mathrm{Hz}$ for digital analysis using our GLP-compliant data acquisition system. ${ }^{17}$

To determine the hemodynamic values at baseline and during Symphony support, the carotid artery flow and pressure waveforms were recorded to derive the mean, peak antegrade, and peak retrograde carotid artery flow and systolic and diastolic arterial pressures proximal and distal to the anastomosis. The hemodynamic values were calculated on a beat-tobeat basis for each 30-s data epoch using the Hemodynamic Evaluation and Assessment Research Tool program ${ }^{18}$ developed in MATLAB (Mathworks, Inc, Natick, Mass). All analyzed beats in each data set (approximately $30-40$ beats $/ 30$-s data set) were averaged to obtain a single representative mean value.

\section{Blood Collection}

Whole blood (approximately $8 \mathrm{~mL}$ ) was collected at baseline and prior to termination after the planned duration of support. From each sample, a complete blood count was measured to obtain the platelet count, and PRP was prepared for platelet aggregation studies. Platelet-poor plasma was snap-frozen with liquid nitrogen for wet immunoblotting of plasma vWF and ADAMTS-13 and determination of ADAMTS-13 activity using FRET. 


\section{Platelet Aggregation}

Fresh whole blood was anticoagulated in $4.5 \mathrm{~mL}$ buffered sodium citrate blood tubes (BD Biosciences, Franklin Lakes, NJ). The blood was transferred to plastic cuvettes and centrifuged at $210 \mathrm{~g}$ for 12 minutes at $22^{\circ} \mathrm{C}$. The supernatant PRP fraction was aspirated into a fresh tube. The remaining blood was centrifuged again at $1000 \mathrm{~g}$ for 15 minutes to sediment platelets. The supernatant platelet-poor plasma was transferred to a fresh tube and snap-frozen for subsequent immunoblotting and FRET analysis.

Platelet aggregation was measured with a 4-channel platelet aggregometer (Chrono-Log Corp, Havertown, Pa). ${ }^{19}$ Each PRP sample (approximately $2.5 \times 10^{8}$ platelets $/ \mathrm{mL}$ ) was incubated with $2 \mathrm{mM}$ calcium at $37^{\circ} \mathrm{C}$ with continuous stirring at $1000 \mathrm{rpm}$. PRP samples were stimulated to aggregate separately with adenosine $5^{\prime}$-diphosphate (ADP) $(10 \mu \mathrm{M})$, collagen $(10 \mu \mathrm{g} / \mathrm{mL})$, and ristocetin $(4 \mathrm{mg} / \mathrm{mL})$. Aggregation was measured at a fixed interval of 10 minutes for ADP and ristocetin and 20 minutes for collagen. The percentage of aggregation was measured as absorbance.

Aggregated PRP was fixed in 10\% buffered formalin. Platelets were stained with fluorescein isothiocyanate (FITC)-conjugated wheatgerm agglutinin for sialic acid in platelet membranes and counterstained with DAPI $^{20}$ to confirm that the aggregates were composed of platelets alone and not nucleated cells.

\section{vWF Analysis}

The relative size of plasma vWF multimers was analyzed by standard immunoblotting techniques. ${ }^{13}$ Plasma samples at baseline and after Symphony support were each combined 1:10 with loading buffer (10 mM Tris $\mathrm{HCl}, 1 \mathrm{mM}$ ethylenediaminetetra-acetic acid, $2 \%$ sodium dodecyl sulfate [SDS], $8 \mathrm{M}$ urea, and bromophenyl blue; $\mathrm{pH}$ 8.0). The samples were heated in a $60^{\circ} \mathrm{C}$ water bath for 20 minutes and loaded into $1.8 \%$ agarose-SDS gels $(7 \times 8.3 \mathrm{~cm} \times 1.5 \mathrm{~mm}, 1 \%$ SDS, $0.375 \mathrm{M}$ Tris $\mathrm{HCl}$ ) and underwent electrophoresis at $40 \mathrm{~V}$ for 4.5 to 5 hours at $4^{\circ} \mathrm{C}$ in Tris buffer $(0.05 \mathrm{M}, 0.384 \mathrm{M}$ glycine, $0.1 \%$ SDS $)$. Proteins were transferred at $30 \mathrm{~V}$ for 16 hours to an Immobilon-P polyvinylidene diflouride membrane (Millipore, Billerica, Mass) by wet electroblotting in phosphate buffer $(0.2 \mathrm{M}$ phosphate, $0.04 \% \mathrm{SDS}, \mathrm{pH} 7.4)$. The blots were incubated with rabbit anti-human vWF primary antibody (1/500; Dako, Inc, Carpinteria, Calif) overnight at $4{ }^{\circ} \mathrm{C}$ and goat anti-rabbit IgG horseradish peroxidase-conjugated secondary antibody (1/3000; Cell Signaling, Inc, Danvers, Mass) for 1 hour. The blots were developed with ECL Plus Western blotting detection reagents (GE Healthcare, Inc, Piscataway, NJ), scanned, and imaged with a Typhoon 9400 Variable Mode Imager (GE Healthcare, Inc).

\section{Analysis of ADAMTS-13}

Plasma ADAMTS-13 levels were analyzed using standard immunoblotting techniques. Plasma samples at baseline and after Symphony support were each diluted 1:5, 1:10,1:20, and 1:40 with loading buffer $(10 \mathrm{mM}$ Tris $\mathrm{HCl}, 1 \mathrm{mM}$ ethylenediaminetetra-acetic acid, 2\% SDS, $8 \mathrm{M}$ urea, and bromophenyl blue; $\mathrm{pH} 8.0$ ). The samples were heated in a $95^{\circ} \mathrm{C}$ water bath for 5 minutes, loaded into $7 \%$ polyacrylamide-SDS gels (1.5-mm thick, $10 \%$ SDS, $0.5 \mathrm{M}$ Tris, $10 \%$ ammonium persulfate and $10 \mu \mathrm{L}$ TEMED), and underwent electrophoresis at $60 \mathrm{~V}$ for 7.5 hours at $4{ }^{\circ} \mathrm{C}$ in a Tris running buffer $(0.025 \mathrm{M}, 0.192 \mathrm{M}$ glycine, $0.1 \%$ SDS; $\mathrm{pH}$ 8.4). The proteins were transferred at $30 \mathrm{~V}$ for 12 hours to an Immobilon-P polyvinylidene diflouride (Millipore) membrane by wet electroblotting in Tris buffer ( $0.025 \mathrm{M}, 0.192$ M glycine, $0.04 \%$ SDS, $20 \%$ methanol; $\mathrm{pH} 8.3$ ). Blots were incubated with rabbit anti-human ADAMTS-13 primary antibody (1/1000; Abcam Inc, Cambridge, Mass) overnight at $4{ }^{\circ} \mathrm{C}$ and goat anti-rabbit IgG horseradish peroxidase-conjugated secondary antibody (1/3000; Cell Signaling, Inc) for 1 hour. Blots were developed with ECL Plus Western blotting detection reagents (GE Healthcare, Inc), scanned, and imaged with a Typhoon 9400 Variable Mode Imager (GE Healthcare, Inc).

ADAMTS-13 activity in plasma was determined with a commercial FRET kit (American Diagnostica, Inc, Stamford, Conn) according to manufacturer's instructions. In brief, Alexa-fluorochrome-conjugated recombinant $\mathrm{vWF}$ was added to the plasma samples. At $37^{\circ} \mathrm{C}$, proteolytic cleavage of the vWF-Alexa substrate uncoupled Alexa fluorochromes and resulted in an increase in fluorescence. The increase in fluorescence over time (maximal volume) was measured with a spectrofluorometer. A standard curve was constructed with a known plasma concentration of ADAMTS-13. ADAMTS-13 activity was determined by interpolation of the maximal volume values from the standard curve.

The total protein content was determined in plasma and carotid arterialring culture supernatant with a bicinchoninic acid colorometric assay. In brief, the samples were diluted 1:50 in phosphate-buffered saline. Standard bicinchoninic acid colorometric protein assay reagents (Pierce Chemical Co, Rockford, Ill) and a 96-well microplate reader (Spectramax 190, Molecular Devices, Sunnyvale, Calif) were used. Sample protein concentrations were interpolated from a standard curve generated with known concentrations of bovine serum albumin (Fisher Scientific, Hampton, NH).

\section{Immunohistochemistry}

At termination, arterial cross sections were harvested near the anastomosis and from the contralateral (control) carotid artery. The tissue sections were embedded in paraffin, sectioned at $4 \mu \mathrm{m}$, deparaffinized, and rehydrated. Standard antigen retrieval was performed. The sections were permeabilized $(0.05 \%$ Saponin), blocked $(2 \%$ bovine serum albumin and $2 \%$ goat serum in phosphate-buffered saline), incubated with rabbit anti-human ADAMTS-13 primary antibody (1/500; Abcam, Inc) for 24 hours at $4^{\circ} \mathrm{C}$ and FITC-conjugated goat anti-rabbit IgG secondary antibody (1/200; Invitrogen, Carlsbad, Calif) for 1 hour at room temperature. The stained sections were mounted with DAPI. Images were acquired using epifluorescence microscopy (Nikon TE2000; Nikon Corp, Tokyo, Japan).

\section{In Vitro Carotid Arterial Ring Culture}

At termination, with sterile technique, carotid artery from within $2 \mathrm{~cm}$ of the anastomosis $(\mathrm{n}=5)$ toward the heart and the contralateral (control) carotid artery were harvested. Carotid arterial rings $(\sim 2 \mathrm{~mm})$ were embedded in Matrigel (BD Biosciences, Bedford, Mass) and mixed 1:1 with endothelial media (Dulbecco's modified Eagles medium, 10\% fetal bovine serum, $1 \%$ penicillin/streptomycin, $150 \mu \mathrm{g} / \mathrm{mL}$ vascular endothelial growth factor, $60 \mathrm{U} / \mathrm{mL}$ heparin). After 36 hours of growth, the supernatant was snap-frozen in liquid nitrogen. Supernatant ADAMTS- 13 protein and activity were determined with immunoblotting and FRET, as previously described.

\section{Enzyme-Linked Immunosorbent Assay}

The plasma IL-6 levels were measured with a sandwich enzyme-linked immunosorbent assay IL-6 kit (Pierce Biotechnology) according to the manufacturer's instructions. In brief, the microtiter plate was precoated with monoclonal antibody specific to bovine IL-6. The plasma samples were incubated in the coated wells overnight at $4{ }^{\circ} \mathrm{C}$. The wells were emptied, washed, and incubated for 1 hour at room temperature with a biotin-conjugated polyclonal antibody preparation specific for IL-6. Microplate wells were aspirated and washed 3 times before incubation with a solution of avidin-conjugated horseradish peroxidase for 30 minutes at room temperature. The washing was repeated. Tetramethylbenzidine was added, and the reaction was incubated for 20 minutes. The reaction was stopped with $1 \mathrm{M} \mathrm{H} 2 \mathrm{~S} 04$, and the signal was measured using a colorimetric microplate reader (Synergy Mx Microplate Reader; Biotek, Winooski, Vt) at $450 \mathrm{~nm}$.

\section{Statistical Analysis}

GraphPad, version 5.00 (Prism, GraphPad Software, Inc, La Jolla, Calif) was used to perform the statistical analyses and plot data. Paired Student's $t$ tests were used to compare the baseline and Symphony hemodynamic 
values, platelet aggregation values, endothelial ADAMTS-13 immunohistochemistry, plasma and cultured carotid arterial-ring supernatant ADAMTS-13 activity, and plasma IL- 6 levels. $P<.05$ (95\% confidence) was considered statistically significant. All data are presented as the mean \pm standard error.

\section{RESULTS \\ Hemodynamics}

The Symphony pump significantly altered the profile of carotid artery hemodynamics (Figure 1). Carotid arterial blood pressure changed from approximately $90 / 50 \mathrm{~mm}$ $\mathrm{Hg}$ at baseline to 200/15 mm Hg during Symphony support (Figure 1, D). Blood pressure values were similar proximal and distal to the anastomosis. During ejection of the pump during native cardiac diastole, approximately $33 \%$ of the blood volume flowed cephalad through the distal carotid artery. In contrast, $67 \%$ of the ejected blood volume flowed retrograde through the proximal carotid artery into the ascending aorta. Diastolic augmentation and afterload reduction were achieved (Figure 1, D, right panels). As previously reported, the Symphony pump augments cardiac output and coronary blood flow and reduces the left ventricular peak systolic pressure, left ventricular external work, and myocardial oxygen consumption. ${ }^{14,21-23}$

Proximal to the anastomosis, the peak antegrade blood flow increased from a baseline flow of $1.4 \pm 0.3$ to $4.3 \pm$ $0.3 \mathrm{~L} / \mathrm{min}$ during pump filling $(P<.001$; Figure $1, D$ and $F)$. The peak flow during pump ejection reversed completely from an antegrade flow of $0.3 \pm 0.1 \mathrm{~L} / \mathrm{min}$ to a retrograde flow of $4.2 \pm 0.4 \mathrm{~L} / \mathrm{min}(P<.001$; Figure $1, D$ and $F)$. Flow reversal in the proximal carotid artery during diastole resulted in antegrade flow augmentation into the aorta.

Distal to the anastomosis, the peak antegrade blood flow increased from a baseline value of $1.1 \pm 0.1 \mathrm{~L} / \mathrm{min}$ to $2.2 \pm$ $0.2 \mathrm{~L} / \mathrm{min}$ during pump ejection $(P=.01$; Figure $1, D$ and $E)$. Flow reversal did not occur in the distal carotid artery.

\section{Platelet Aggregation}

After 7 days of Symphony support, platelet aggregation with ADP and collagen were unchanged. However, ristocetinmediated platelet aggregation through the vWF pathway was significantly impaired (Figure 2, $A-E$ ). The amplitude of aggregation decreased from $20 \% \pm 4 \%$ at baseline to $14 \% \pm 2 \%$ after 1 week of Symphony support $(P<.01)$. The slope of aggregation decreased from $41 \% \pm 4 \% / \mathrm{min}$ to $30 \% \pm 4 \% / \mathrm{min}$ $(P<.05)$. Total platelet aggregation, as determined by the area under the aggregation curve, decreased from $335 \% \pm 20 \% /$ $\min$ to $257 \% \pm 28 \% / \mathrm{min}(P<.001)$. The platelet number from the complete blood count did not change significantly from $251,000 \pm 19,000$ platelets $/ \mu \mathrm{L}$ at baseline to 258,000 $\pm 17,000$ platelets $/ \mu \mathrm{L}$ after Symphony support.

Morphologic differences existed between the platelet aggregation curves at baseline and during Symphony support (Figure 2,D). The baseline curves demonstrated a biphasic aggregation pattern in which the initial robust phase was followed by a smaller secondary phase of aggregation. After peak aggregation, the aggregates were stable and did not dissociate. In contrast, after Symphony support, a biphasic response was absent, and the aggregates spontaneously dissociated at a slow rate. Similarly, after Symphony support, the ristocetin-induced platelet aggregates were smaller and less numerous than the aggregates at baseline (Figure 2,E).

\section{Plasma vWF Immunoblotting}

In 11 of 14 calves, wet immunoblotting demonstrated a modest reduction in the high-molecular-weight vWF multimers (Figure 2, F). A shift toward greater numbers of mid- and low-molecular-weight vWF multimers was evident.

\section{Plasma ADAMTS-13 Protein and Activity}

In 10 of 14 calves, wet immunoblotting demonstrated an increase in plasma ADAMTS-13 protein (Figure 3, $A$ and $B)$. In 11 of 14 animals, the plasma ADAMTS-13 activity increased. ADAMTS-13 activity after Symphony support increased by $24.7 \pm 10.1 \mathrm{ng} / \mathrm{mL}(P<.05$; Figure $3, C)$. Total plasma protein was not different from baseline after Symphony support.

\section{Carotid Artery ADAMTS-13 Immunohistochemistry}

The carotid artery endothelium near the anastomosis exhibited decreased intracellular ADAMTS-13 levels (Figure 4). The staining area was $23.5 \% \pm 0.4 \%$ in the control carotid artery and $22.6 \% \pm 0.5 \%$ near the anastomosis $(P=.07)$. The staining intensity was $7.1 \pm 0.3$ arbitrary units in the control carotid artery and $6.1 \pm 0.3$ arbitrary units near the anastomosis $(P<.05$; Figure $4, A-H)$.

\section{Cultured Carotid Artery ADAMTS-13 Activity}

In all cultured carotid arteries, wet immunoblotting of the supernatant demonstrated an increase in plasma ADAMTS13 protein and activity (Figure 5). ADAMTS-13 activity was $39.7 \% \pm 10.7 \%$ greater in the supernatant from the carotid artery near the anastomosis compared with that in the control carotid artery $(P<.05$; Figure $5, C)$. The total supernatant protein was not different between the control carotid arteries and carotid arteries near the anastomosis.

\section{Plasma IL-6}

At baseline, the plasma IL- 6 was $1.04 \pm 0.74 \mathrm{ng} / \mathrm{mL}$. After 1 week of Symphony support, the plasma IL-6 was $0.89 \pm 0.55 \mathrm{ng} / \mathrm{mL}$. This change was not statistically significant $(P=.49)$.

\section{DISCUSSION}

Our findings suggest potential in vivo mechanistic pathways of vWF degradation during mechanical circulatory 

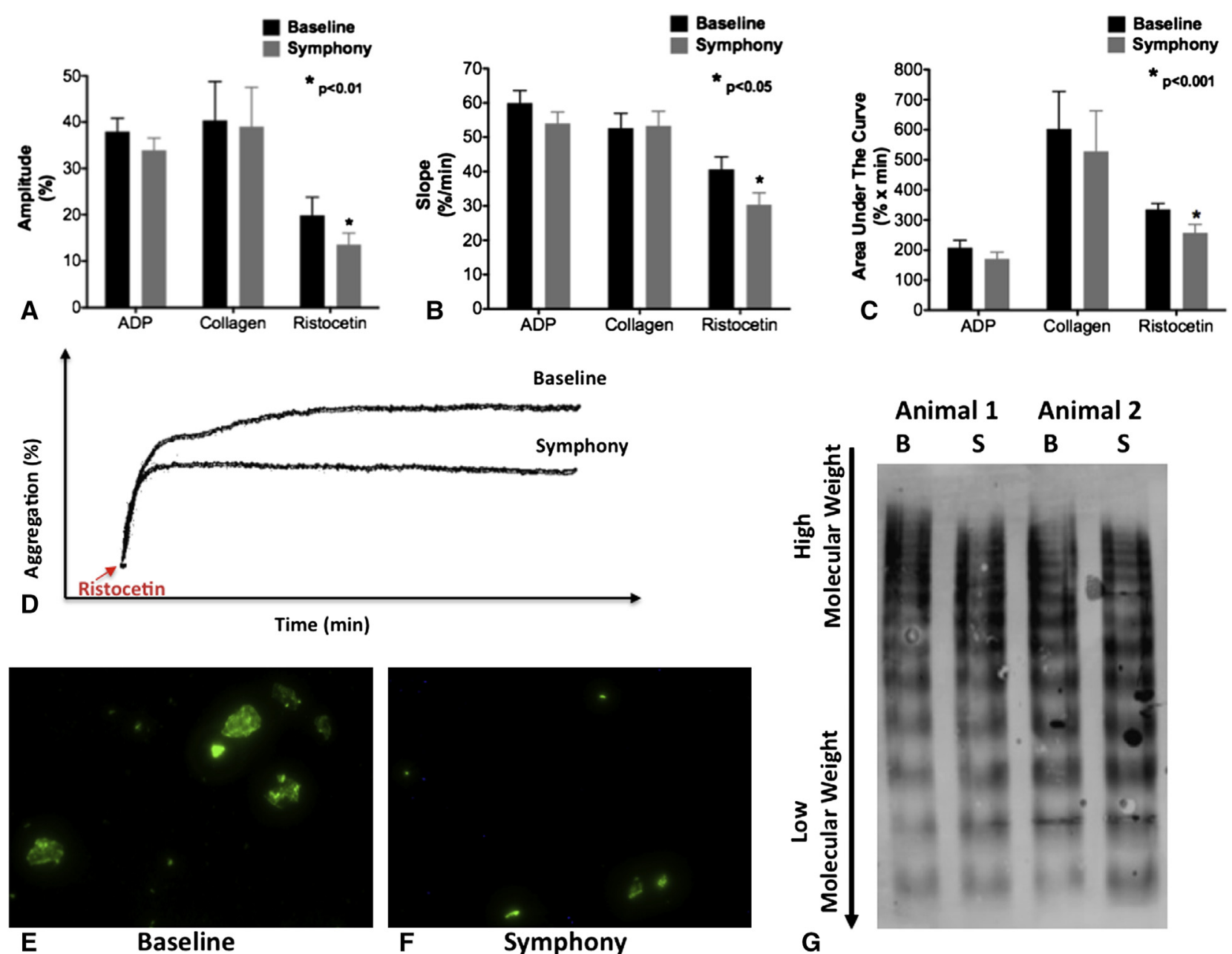

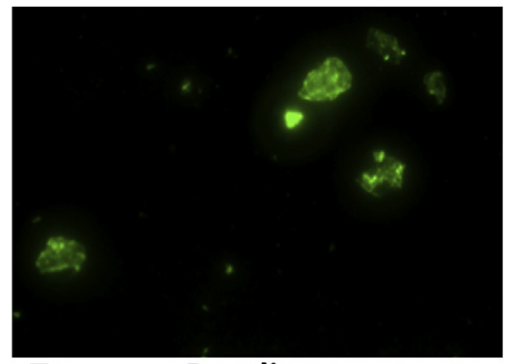

E Baseline

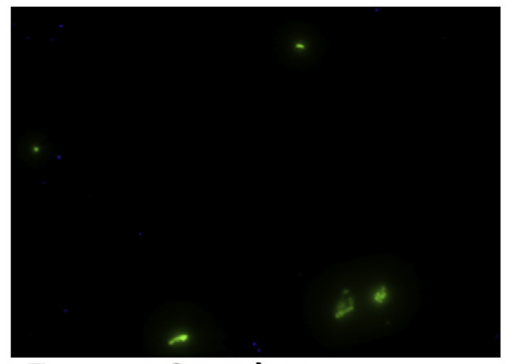

F Symphony
G

FIGURE 2. A-C, Platelet aggregometry with adenosine $5^{\prime}$-diphosphate $(A D P)$, collagen, and ristocetin demonstrated impaired aggregation through the ristocetin-von Willebrand factor $(v W F)$ pathway but not through the ADP or collagen pathways. D, Example platelet aggregation curves at baseline and after Symphony support demonstrated impaired platelet aggregation through the vWF pathway. Morphologic differences existed between the platelet aggregation curves. The baseline curve demonstrated a biphasic aggregation pattern in which the initial robust phase was followed by a smaller secondary phase of aggregation. After peak aggregation, the aggregates were stable and did not dissociate. In contrast, after Symphony support, a biphasic response was absent, and the aggregates spontaneously dissociated at a slow rate. E and F, Similarly, after Symphony support, the ristocetin-induced platelet aggregates were smaller and less numerous than the aggregates at baseline. This finding may be related to the loss of the secondary phase of the biphasic platelet aggregation response. G, After Symphony support, agarose-SDS gel electrophoresis and wet immunoblotting demonstrated a mild reduction of the highest molecular-weight vWF multimers. A shift toward greater numbers of mid- and low-molecular-weight multimers was evident. $B$, Baseline; $S$, Symphony.

support. The Symphony pump significantly altered the hemodynamic profile near the anastomosis. After shortterm support in male calves, we observed: (1) impaired platelet aggregation through the ristocetin-vWF pathway but not the ADP or collagen pathways, (2) modestly decreased high-molecular-weight plasma vWF multimers, (3) modestly increased plasma ADAMTS-13, and (4) increased ADAMTS-13 production by endothelium near the anastomosis. IL-6, which inhibits ADAMTS-13 activity, was unchanged and was unlikely to have played a role in this process. We speculate that an altered hemodynamic profile near the end-to-side anastomosis activated vWF for enhanced cleavage by ADAMTS-13. In parallel, increased endothelial production of ADAMTS-13 may also have increased degradation of vWF. These mechanistic pathways likely contribute to bleeding episodes in patients with an LVAD.

\section{Normal Mechanisms of vWF Degradation: Shear Stress and ADAMTS-13}

vWF, a 500 - to $20,000-\mathrm{kD}$ multimeric glycoprotein, is constitutively manufactured by endothelial cells, circulates in the plasma, and participates in primary hemostasis. ${ }^{24} \mathrm{At}$ sites of endothelial damage, turbulent blood flow and 


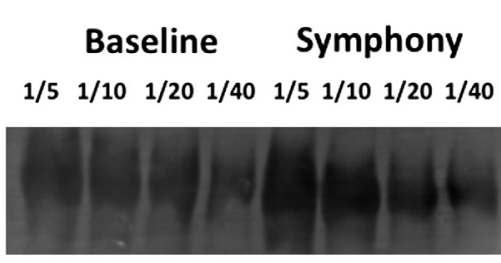

A

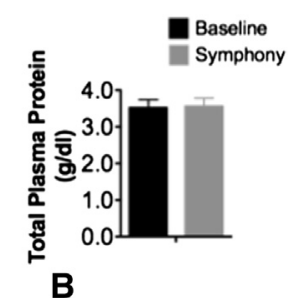

Baseline Symphony ${ }^{*} p<0.05$

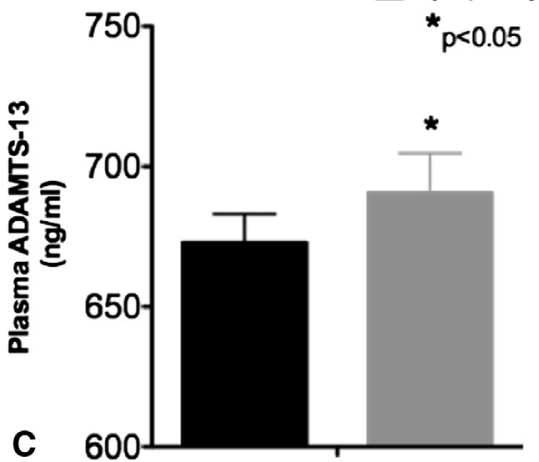

FIGURE 3. A, Wet immunoblotting demonstrated increased plasma ADAMTS-13 protein after Symphony support. B, A bicinchoninic acid colorometric assay demonstrated that the total plasma protein was not different from baseline after Symphony support. C, ADAMTS-13 measured with fluorescent resonance energy transfer demonstrated increased plasma ADAMTS-13 activity.

altered shear stress cause vWF to undergo a conformational change that facilitates vWF tethering to subendothelial collagen and platelets by way of the glycoprotein $1 \mathrm{~b}-\mathrm{V}$-IX complex. ${ }^{25}$ Thus, a platelet plug covers the breached endothelium. During this process, the highest molecular weight $\mathrm{vWF}$ multimers are most susceptible to activation by shear stress. However, shear-induced activation also renders $\mathrm{vWF}$ more sensitive to proteolytic cleavage by ADAMTS- $13,{ }^{26}$ the vWF-specific plasma metalloprotease. As such, increased proteolytic cleavage of vWF can be observed with an increase in shear-induced conformational activation of vWF, an increase in ADAMTS-13, or both.

The basic mechanism of shear stress-induced activation and degradation of vWF is well described. ${ }^{24}$ Clinical correlates are well documented. For example, in patients with critical aortic stenosis, shear stress through the stenotic aortic annulus correlates with loss of high-molecularweight vWF multimers. ${ }^{27}$ Within hours of aortic valve replacement surgery (and re-establishment of a normal shear stress profile), high-molecular-weight vWF multimers return. These findings highlight in humans the mechanistic importance of shear stress-induced activation and degradation of vWF.

Importantly, LVADs alter shear stress. ${ }^{28}$ The blood of a patient with an LVAD is continuously exposed to supraphysiologic shear stresses, especially near the end-to-side anastomosis. As a result, pathologic von Willebrand degradation occurs in patients with continuous-flow ${ }^{1-11}$ and pulsatile-flow ${ }^{1,4,5,9}$ LVADs. It has been speculated that acquired von Willebrand syndrome in LVAD patients develops from the loss of high-molecular-weight vWF due to mechanical destruction and proteolysis by ADAMTS-13. ${ }^{29}$ Experimental evidence supports the hypothesis that the major mechanism of vWF degradation is mechanical destruction of vWF, with ADAMTS-13 playing a smaller role. ${ }^{13}$ Our current data further suggest that ADAMTS-13 plays an ancillary role in this process. Likely, shear-induced activation of vWF results in enhanced vWF cleavage by ADAMTS- 13 .

ADAMTS-13 in the plasma and supernatant from the cultured carotid artery rings near the anastomosis increased modestly. These findings suggest that altered hemodynamics near the end-to-side anastomosis activated endothelium to increase production of ADAMTS-13. Evidence has suggested that abnormal stresses stimulate endothelial production $^{30}$ and increased activity ${ }^{31}$ of ADAMTS-13. Additionally, in a previous study in our model, circulatory support triggered phenotypic changes in the endothelium that resulted in a hypermetabolic state ${ }^{32}$ that may have enhanced the production of ADAMTS-13. Therefore, we speculate that non-physiologic hemodynamic loading (increased pressure, increased flow, reverse flow, turbulence, changes in shear stress) of endothelial cells near the anastomosis increased production of ADAMTS- 13 .

There may have been multiple sources of increased plasma ADAMTS-13 protein. ADAMTS-13 is produced by hepatic stellate cells, ${ }^{33}$ endothelium, ${ }^{34}$ and platelets. ${ }^{35}$ In vitro mechanical circulatory support leads to platelet activation and release of ADAMTS-13 from platelets into the plasma. ${ }^{13}$ Therefore, the increase in plasma ADAMTS-13 that we observed was likely from a combination of activated platelets, hypermetabolic endothelium near the anastomosis, and/or other sources.

Our results were not due to a change in the plasma IL-6 concentration. IL-6 inhibits ADAMTS-13 activity. ${ }^{36}$ As such, changes in the inflammatory milieu caused by continuous blood contact with the interior artificial surface of the pump may have influenced IL-6 levels and altered ADAMTS-13 activity. However, the plasma IL-6 levels did not change significantly and were unlikely to have accounted for the observed changes in ADAMTS-13 activity. Other cytokines such as tumor necrosis factor- $\alpha$ and IL-10 do not influence ADAMTS-13 activity ${ }^{36}$ and also likely did not influence $\mathrm{vWF}$ degradation. Together, these findings suggest that acquired von Willebrand syndrome in patients with an LVAD is independent of the systemic cytokine profile.

\section{Clinical Implications}

Multiple LVADs are clinically available or poised for clinical trials. ${ }^{37}$ Each device produces a unique 

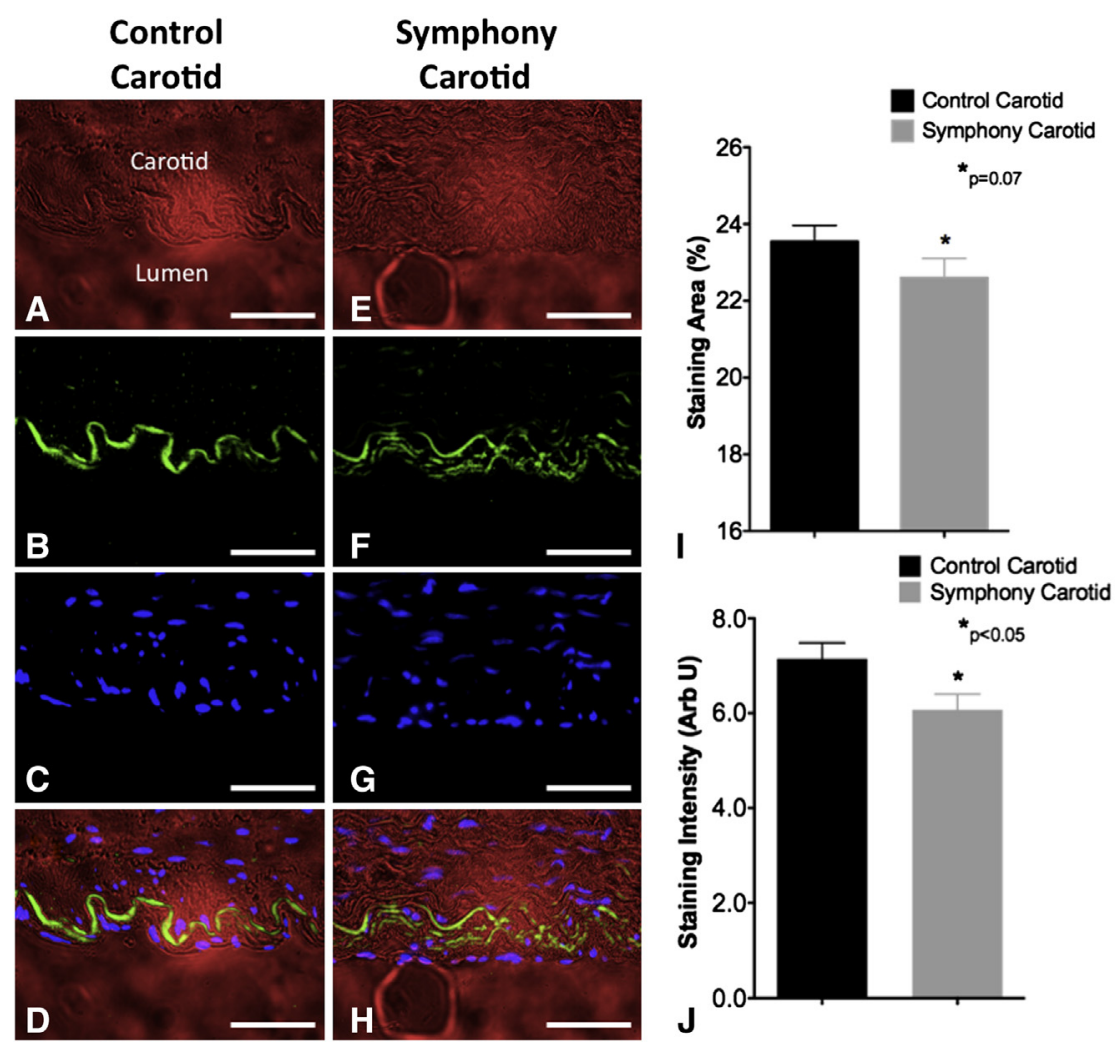

FIGURE 4. ADAMTS-13 immunohistochemical staining demonstrated that endothelial cells contained reduced ADAMTS-13 near the anastomosis compared with the contralateral (control) carotid arteries. A-H, The staining pattern of ADAMTS-13 in the control carotid artery was nearly uniform and followed the contours of the endothelium. However, endothelial ADAMTS-13 near the anastomosis demonstrated diffuse staining that suggested active mobilization of ADAMTS-13. A and E, Bright field images; B and F, FITC-conjugated ADAMTS-13 immunohistochemical staining; C and G, DAPI conuclear stain; D and H, Overlay $(40 \times$, white bar $=100 \mu \mathrm{m})$; and I and J, Total staining area and staining intensity for ADAMTS-13 decreased in endothelium near the anastomosis compared with the controls. Arb $U$, Arbitrary units.

hemodynamic profile with varying pulsatility, turbulence, and shear stress, which serve as important biomechanical activators of the clotting system. ${ }^{38}$ As such, certain device types may trigger more pronounced activation and degradation of vWF. For example, quantitative and qualitative changes in shear stress may have important clinical implications. Pulsatile-flow and continuous-flow LVADs alter the shear stress profile differently. Pulsatile pumps, such as the Symphony pump, alter the shear stress gradient - rapid acceleration of blood during filling and emptying of the pump produces bursts of high and low shear. In contrast, during support with a continuousflow LVAD, native pulsatility is greatly diminished. ${ }^{39}$ As a result, continuous nonphysiologically high shear stress persists, with little variation in the magnitude of the shear stress.

Strong clinical evidence has shown that continuous-flow LVADs produce more severe impairment of $\mathrm{vWF}$ and more frequent bleeding episodes than pulsatile pumps. ${ }^{40}$ In light of our data, we speculate that a high shear stress gradient with a pulsatile-flow LVAD is less traumatic to vWF than the constant high shear stress of a continuous-flow device.
Our vWF immunoblotting analysis demonstrated mild reductions of the highest molecular weight vWF multimers that were less dramatic than with continuous-flow LVADs. ${ }^{1,3,5,6,8,9}$ Differences between pulsatile-flow and continuous-flow LVAD activation and degradation of vWF warrant additional investigation.

Interestingly, and in additional support of this point, pathologic degradation of vWF has not been observed in patients with a total artificial heart. ${ }^{1}$ Pulsatile blood flow through a large end-to-end anastomosis may reproduce a normal shear stress profile without the turbulence and shear stress alterations that occur at an end-to-side anastomosis. Hemodynamically, the end-to-end configuration of a total artificial heart recapitulates normal cardiovascular anatomy and produces physiologic flow patterns in series with the circulation. In contrast, the end-to-side configuration of an LVAD introduces a second circuit in parallel with the circulation that causes flow channels to merge at the site of the anastomosis. The effect is turbulence, high shear stress, and high shear gradients at the end-to-side anastomosis. Interestingly, vWF degradation occurs if the device is connected end-to-side to the aorta or the 


\section{Control Symphony Carotid Carotid}

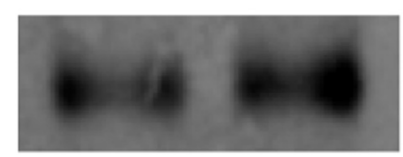

A

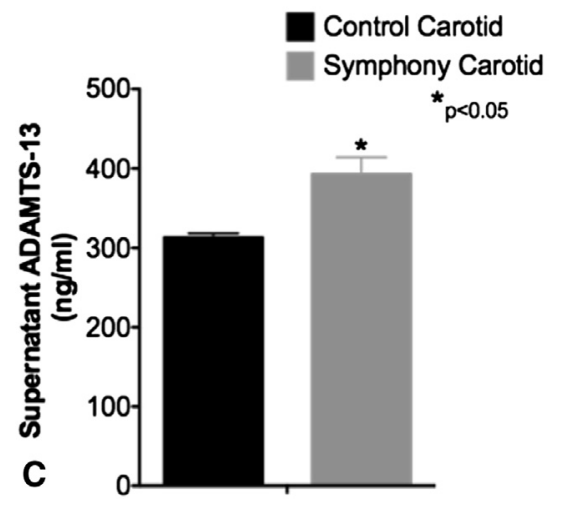

FIGURE 5. A, Wet immunoblotting demonstrated increased ADAMTS13 protein in supernatant from cultured carotid artery near the anastomosis compared with the contralateral (control) carotid. B, A bicinchoninic acid colorometric assay demonstrated that the total supernatant protein was not different between the control carotid artery and the carotid artery near the anastomosis. C, ADAMTS-13 measured with fluorescent resonance energy transfer demonstrated increased ADAMTS-13 activity in supernatant from the carotid artery near the anastomosis compared with the controls.

subclavian artery. ${ }^{11}$ Therefore, we speculate that it is not the site of the anastomosis, but rather the flow patterns at the end-to-side anastomosis that may have important implications for vWF degradation and the design of next generation mechanical circulatory support devices. As such, the end-to-side anastomosis should be considered as a target for therapies.

The role of ADAMTS-13 in LVAD-induced acquired von Willebrand syndrome is unclear. The limited existing data are conflicting. In our study, with paired baseline controls, we observed a small $(24.7 \pm 10.1 \mathrm{ng} / \mathrm{mL})$, but statistically, significant increase in plasma ADAMTS-13 activity. The clinical relevance of such a modest increase is unknown. Clinical data have suggested that ADAMTS-13 does not play a major role in acquired von Willebrand syndrome during mechanical circulatory support. ${ }^{3,4,8}$ However, these studies were conducted in small subgroups of patients, lacked controls, and it was not possible to make definitive conclusions. ${ }^{13}$ In vitro data suggest that ADAMTS-13 plays at least a minor role in LVAD-induced von Willebrand syndrome but was unlikely to be the dominant mechanism. ${ }^{13}$ The effects of mechanical circulatory support on ADAMTS-13 protein and activity remain to be determined in a large cohort of patients with appropriate paired controls. In these studies, it will be necessary to measure plasma ADAMTS-13 protein and activity, which may be enhanced by shear stress. $^{26}$

Mild impairment of prothrombotic pathways represents a potential clinical benefit to prevent thromboembolism in a patient with an LVAD. However, the optimal level of vWF impairment is unknown. In humans, $>50 \% \mathrm{vWF}$ deficiency is suggestive (not diagnostic) of von Willebrand syndrome, but rarely causes bleeding. ${ }^{41}$ With the Symphony pump, we observed a $30 \%$ functional impairment of vWF-platelet aggregation, which may not be of clinical concern. Similarly, the intra-aortic balloon pump, a device which produces a similar hemodynamic profile to the Symphony pump, has not been shown to produce acquired von Willebrand syndrome, and bleeding occurs in only $6 \%$ of intra-aortic balloon pump patients ${ }^{42}$ versus $27 \%$ to $75 \%$ of patients with an LVAD. ${ }^{2-4,7,8}$ An ongoing clinical trial will determine the risk of gastrointestinal bleeding with the Symphony pump. Less vWF degradation compared to continuous-flow LVADs may result in a lower incidence of bleeding in patients with the Symphony pump.

\section{Study Limitations}

Patients with heart failure typically develop endothelial cell dysfunction, which was not present in the normal calves used in the present study. Therefore, our results should be interpreted in light of this limitation and should not be extrapolated to human patients without caution.

\section{CONCLUSIONS}

Our findings suggest in vivo mechanistic pathways of vWF degradation during mechanical circulatory support. Nonphysiologic hemodynamics near the anastomosis increased local endothelial production of ADAMTS-13. Hemodynamic activation of vWF and increased plasma ADAMTS-13 may have contributed to reduced high-molecular-weight vWF multimers and impairment of the vWF-platelet aggregation pathway. Platelet aggregation with ADP and collagen was unchanged, as were the IL-6 levels. Further delineation of these pathways may be of significance for the field of mechanical circulatory support.

The authors acknowledge and thank the University of Louisville veterinary staff, as well as Dr Sumanth Prabhu, Dr Syed Khundmiri, Mary Anne Hauck, and Abiomed, Inc, for their assistance.

\section{References}

1. Heilmann C, Geisen U, Beyersdorf F, Nakamura L, Benk C, Berchtold-Herz M, et al. Acquired von Willebrand syndrome in patients with ventricular assist device or total artificial heart. Thromb Haemost. 2010;103:962-7.

2. Uriel N, Pak SW, Jorde UP, Jude B, Susen S, Vincentelli A, et al. Acquired von Willebrand syndrome after continuous-flow mechanical device support 
contributes to a high prevalence of bleeding during long-term support and at the time of transplantation. J Am Coll Cardiol. 2010;56:1207-13.

3. Klovaite J, Gustafsson F, Mortensen SA, Sander K, Nielsen LB. Severely impaired von Willebrand factor-dependent platelet aggregation in patients with a continuous-flow left ventricular assist device (HeartMate II). J Am Coll Cardiol. 2009;53:2162-7.

4. Crow S, Milano C, Joyce L, Chen D, Arepally G, Bowles D, et al. Comparative analysis of von Willebrand factor profiles in pulsatile and continuous left ventricular assist device recipients. ASAIO J. 2010;56:441-5.

5. Geisen U, Heilmann C, Beyersdorf F, Benk C, Berchtold-Herz M, Schlensak C, et al. Non-surgical bleeding in patients with ventricular assist devices could be explained by acquired von Willebrand disease. Eur J Cardiothorac Surg. 2008;33:679-84.

6. Steinlechner B, Dworschak M, Birkenberg B, Duris M, Zeidler P, Fischer H, et al. Platelet dysfunction in outpatients with left ventricular assist devices. Ann Thorac Surg. 2009;87:131-7.

7. Crow S, Chen D, Milano C, Thomas W, Joyce L, Piacentino V III, et al. Acquired von Willebrand syndrome in continuous-flow ventricular assist device recipients. Ann Thorac Surg. 2010;90:1263-9; discussion 9.

8. Meyer AL, Malehsa D, Bara C, Budde U, Slaughter MS, Haverich A, et al. Acquired von Willebrand syndrome in patients with an axial flow left ventricular assist device. Circ Heart Fail. 2010;3:675-81.

9. Heilmann C, Geisen U, Beyersdorf F, Nakamura L, Trummer G, BerchtoldHerz M, et al. Acquired von Willebrand syndrome is an early-onset problem in ventricular assist device patients. Eur J Cardiothorac Surg. 2011;40: 1328-33; discussion 233.

10. Heilmann C, Geisen U, Beyersdorf F, Nakamura L, Benk C, Trummer G, et al. Acquired von Willebrand syndrome in patients with extracorporeal life support (ECLS). Intensive Care Med. 2012;38:62-8.

11. Goda M, Jacobs S, Rega F, Peerlinck K, Jacquemin M, Droogne W, et al. Time course of acquired von Willebrand disease associated with two types of continuous-flow left ventricular assist devices: HeartMate II and CircuLite Synergy Pocket Micro-pump. J Heart Lung Transplant. 2013;32:539-45.

12. Islam S, Cevik C, Madonna R, Frandah W, Islam E, Islam S, et al. Left ventricular assist devices and gastrointestinal bleeding: a narrative review of case reports and case series. Clin Cardiol. 2013;36:190-200.

13. Dassanayaka S, Slaughter MS, Bartoli CR. Mechanistic pathway(s) of acquired von Willebrand syndrome with a continuous-flow ventricular assist device: in vitro findings. ASAIO J. 2013;59:123-9.

14. Bartoli CR, Wilson GC, Giridharan GA, Slaughter MS, Sherwood LC, Spence PA, et al. A novel subcutaneous counterpulsation device: acute hemodynamic efficacy during pharmacologically induced hypertension, hypotension, and heart failure. Artif Organs. 2010;34:537-45.

15. ClinicalTrials. Symphony: the implantable counter pulsation device (CPD) safety and feasibility trial. Available at: http://www.clinicaltrials.gov/ct2/show/ NCT01543022?term =symphony\&rank=1. Accessed May 15, 2013.

16. Bartoli CR, Dowling RD, Wilson GC, Giridharan GA, Slaughter MS, Sherwood LC, et al. Response to letter to the editor: a novel subcutaneous counterpulsation device: acute hemodynamic efficacy during pharmacologically induced hypertension, hypotension, and heart failure. Artif Organs. 2011;35: 93-5.

17. Koenig SC, Woolard C, Drew G, Unger L, Gillars K, Ewert D, et al. Integrated data acquisition system for medical device testing and physiology research in compliance with good laboratory practices. Biomed Instrum Technol. 2004;38: 229-40.

18. Schroeder MJ, Perreault B, Ewert DL, Koenig SC. HEART: an automated beatto-beat cardiovascular analysis package using MATLAB. Comput Biol Med. 2004;34:371-88.

19. Sithu SD, Srivastava S, Siddiqui MA, Vladykovskaya E, Riggs DW, Conklin DJ, et al. Exposure to acrolein by inhalation causes platelet activation. Toxicol Appl Pharmacol. 2010;248:100-10.

20. Bartoli CR, Wead WB, Giridharan GA, Prabhu SD, Koenig SC, Dowling RD. Mechanism of myocardial ischemia with an anomalous left coronary artery from the right sinus of Valsalva. J Thorac Cardiovasc Surg. 2012;144: 402-8.
21. Koenig SC, Pantalos GM, Litwak KN, Gillars KJ, Giridharan GA, Maguire M et al. Hemodynamic and left ventricular pressure-volume responses to counterpulsation in mock circulation and acute large animal models. Conf Proc IEEE Eng Med Biol Soc. 2004;5:3761-4.

22. Koenig SC, Spence PA, Pantalos GM, Dowling RD, Litwak KN. Development and early testing of a simple subcutaneous counterpulsation device. ASAIO J. 2006;52:362-7.

23. Koenig SC, Litwak KN, Giridharan GA, Pantalos GM, Dowling RD, Prabhu SD et al. Acute hemodynamic efficacy of a 32-ml subcutaneous counterpulsation device in a calf model of diminished cardiac function. ASAIO J. 2008;54:578-84.

24. Sadler JE. Biochemistry and genetics of von Willebrand factor. Annu Rev Biochem. 1998;67:395-424.

25. Siedlecki CA, Lestini BJ, Kottke-Marchant KK, Eppell SJ, Wilson DL, Marchant RE. Shear-dependent changes in the three-dimensional structure of human von Willebrand factor. Blood. 1996;88:2939-50.

26. Tsai HM, Sussman II, Nagel RL. Shear stress enhances the proteolysis of von Willebrand factor in normal plasma. Blood. 1994;83:2171-9.

27. Vincentelli A, Susen S, Le Tourneau T, Six I, Fabre O, Juthier F, et al. Acquired von Willebrand syndrome in aortic stenosis. N Engl J Med. 2003; 349:343-9.

28. Fraser KH, Zhang T, Taskin ME, Griffith BP, Wu ZJ. A quantitative comparison of mechanical blood damage parameters in rotary ventricular assist devices: shear stress, exposure time and hemolysis index. J Biomech Eng. 2012;134: 081002 .

29. Tsai HM. von Willebrand factor, shear stress, and ADAMTS13 in hemostasis and thrombosis. ASAIO J. 2012;58:163-9.

30. Turner NA, Nolasco L, Ruggeri ZM, Moake JL. Endothelial cell ADAMTS-13 and VWF: production, release, and VWF string cleavage. Blood. 2009;114: $5102-11$.

31. Lo B, Nierich AP, Kalkman CJ, Fijnheer R. Relatively increased von Willebrand factor activity after off-pump coronary artery bypass graft surgery. Thromb Hae most. 2007;97:21-6.

32. Bartoli CR, Spence PA, Koenig SC, Dowling RD. Nonphysiologic blood flow triggers endothelial and arterial remodeling in vivo: implications for novel left ventricular assist devices with a peripheral anastomosis. J Thorac Cardiovasc Surg. December 9, 2013 [Epub ahead of print]

33. Uemura M, Tatsumi K, Matsumoto M, Fujimoto M, Matsuyama T, Ishikawa M, et al. Localization of ADAMTS13 to the stellate cells of human liver. Blood. 2005;106:922-4.

34. Turner N, Nolasco L, Tao Z, Dong JF, Moake J. Human endothelial cells synthesize and release ADAMTS-13. J Thromb Haemost. 2006;4:1396-404.

35. Liu L, Choi H, Bernardo A, Bergeron AL, Nolasco L, Ruan C, et al. Plateletderived VWF-cleaving metalloprotease ADAMTS-13. J Thromb Haemost. 2005;3:2536-44.

36. Bernardo A, Ball C, Nolasco L, Moake JF, Dong JF. Effects of inflammatory cytokines on the release and cleavage of the endothelial cell-derived ultralarge von Willebrand factor multimers under flow. Blood. 2004;104:100-6.

37. Bartoli CR, Dowling RD. The future of adult cardiac assist devices: novel systems and mechanical circulatory support strategies. Cardiol Clin. 2011;29: 559-82.

38. Nesbitt WS, Westein E, Tovar-Lopez FJ, Tolouei E, Mitchell A, Fu J, et al. A shear gradient-dependent platelet aggregation mechanism drives thrombus formation. Nat Med. 2009; 15:665-73.

39. Bartoli CR, Giridharan GA, Litwak KN, Sobieski M, Prabhu SD, Slaughter MS et al. Hemodynamic responses to continuous versus pulsatile mechanical unloading of the failing left ventricle. ASAIO J. 2010;56:410-6.

40. Crow S, John R, Boyle A, Shumway S, Liao K, Colvin-Adams M, et al. Gastrointestinal bleeding rates in recipients of nonpulsatile and pulsatile left ventricular assist devices. J Thorac Cardiovasc Surg. 2009;137:208-15.

41. Torres R, Fedoriw Y. Laboratory testing for von Willebrand disease: toward a mechanism-based classification. Clin Lab Med. 2009;29:193-228.

42. Sjauw KD, Engstrom AE, Vis MM, van der Schaaf RJ, Baan J Jr, Koch KT, et al A systematic review and meta-analysis of intra-aortic balloon pump therapy in ST-elevation myocardial infarction: should we change the guidelines? Eur Heart J. 2009;30:459-68. 